\title{
Fast electron slowing-down and diffusion in a high temperature coronal X-ray source
}

\author{
R. K. Galloway ${ }^{1}$, A. L. MacKinnon ${ }^{2}$, E. P. Kontar ${ }^{3}$, and P. Helander ${ }^{4}$ \\ ${ }^{1}$ Department of Physics and Astronomy, Kelvin Building, University of Glasgow, Glasgow G12 8QQ, UK \\ e-mail: ross@astro.gla.ac.uk \\ 2 Department of Adult and Continuing Education, St. Andrews Building, University of Glasgow, Glasgow G3 6NH, UK \\ e-mail: a.mackinnon@educ.gla.ac.uk \\ ${ }^{3}$ Department of Physics and Astronomy, Kelvin Building, University of Glasgow, Glasgow G12 8QQ, UK \\ e-mail: eduard@astro.gla.ac.uk \\ ${ }^{4}$ UKAEA, Culham Science Centre, Abingdon OX14 3DB, UK \\ e-mail: per.helander@ukaea.org.uk
}

Received 7 October 2004 / Accepted 18 April 2005

\begin{abstract}
Finite thermal velocity modifications to electron slowing-down rates may be important for the deduction of solar flare total electron energy. Here we treat both slowing-down and velocity diffusion of electrons in the corona at flare temperatures, for the case of a simple, spatially homogeneous source. Including velocity diffusion yields a consistent treatment of both "accelerated" and "thermal" electrons. It also emphasises that one may not invoke finite thermal velocity target effects on electron lifetimes without simultaneously treating the contribution to the observed X-ray spectrum from thermal electrons. We present model calculations of the X-ray spectra resulting from injection of a power-law energy distribution of electrons into a source with finite temperature. Reducing the power-law distribution low-energy cutoff to lower and lower energies only increases the relative magnitude of the thermal component of the spectrum, because the lowest energy electrons simply join the background thermal distribution. Acceptable fits to RHESSI flare data are obtained using this model. These also demonstrate, however, that observed spectra may in consequence be acceptably consistent with rather a wide range of injected electron parameters.
\end{abstract}

Key words. Sun: flares - Sun: X-rays, gamma-rays - acceleration of particles - plasmas

\section{Introduction}

$\mathrm{X}$ - and $\gamma$-ray radiations give the most direct window on accelerated electrons in flares. They have revealed that accelerated particles, electrons and/or ions, are an energetically major product of the flare energy release process (e.g. Vilmer \& MacKinnon 2003).

Brown et al. (2003) have emphasised the importance of the source-averaged electron distribution as a useful "halfway house" between the observed photon spectrum and the distribution of electrons initially injected into the source region, i.e. the immediate product of the acceleration process. Assumptions about the dominant factors in electron transport then allow deduction from the source-averaged electron distribution of the distribution output by the acceleration process. Quantities like the total energy released in the form of fast electrons follow immediately.

Brown (1971) first analysed the case in which electrons slow down via Coulomb collisions in a cold target, i.e. a region in which ambient particle thermal speeds are all very much less than those of the X-ray emitting electrons. Key results were given for a photon spectrum $I(\epsilon)$ (photons $\mathrm{cm}^{-2} \mathrm{keV}^{-1} \mathrm{~s}^{-1}$ ) depending on photon energy $\epsilon$ as a power-law, i.e. $I(\epsilon) \sim \epsilon^{-\gamma}$ for some $\gamma>0$. Such a photon spectrum is often observed. Assuming a cold target from which no electrons escape, it implies an injected electron energy distribution depending on electron energy $E$ as $E^{-\gamma-1}$. The total energy content of such a distribution is governed by the lowest electron energy for which this power-law form holds good. Unfortunately observations remain ambiguous on the likely value of this lower cutoff, so the total flare energy in accelerated electrons remains uncertain by more than an order of magnitude. The flare energy in electrons of energies $>25 \mathrm{keV}$ appears to be a large fraction of the total flare energy (Lin \& Hudson 1976; Hoyng et al. 1976; Saint-Hilaire \& Benz 2005); observations even exist suggesting a low energy cutoff in the $2-5 \mathrm{keV}$ range (Kane et al. 1992).

Emslie (2003) has pointed out that the cold target assumption may be invalid for the lowest energy (few keV) accelerated flare electrons. Spatial structure (Emslie et al. 2003) of RHESSI (Reuven Ramaty High Energy Solar Spectroscopic Imager) images in particular suggests that these electrons stop entirely in the corona, in high temperature $\left(>10^{7} \mathrm{~K}\right)$ 
regions. Then the test particle slowing-down rate no longer increases monotonically with decreasing particle energy; as particle speed approaches ambient particle thermal speeds from above, the rate of loss of energy to background particles decreases, exhibiting a zero for a fast particle energy $E_{\text {crit }}$ very close to the ambient electron thermal energy. Emslie contends that this value should be used as the minimum possible lower energy cutoff when evaluating fast electron total injected energy; electrons below this energy do not slow down monotonically, instead merging with the background thermal distribution. Emslie's suggested procedure has been employed by Lin et al. (2003) to estimate the fast electron energy content in the flare of 23rd July 2002.

Emslie's discussion is expressed entirely in terms of the systematic slowing-down rate. This gives valuable insight but cannot give a complete description of the evolution of injected electrons. Suppose we inject a mono-energetic electron distribution at $E_{\text {crit }}$. Clearly, although the systematic slowing-down rate at $E_{\text {crit }}$ is zero, the injected electrons will not stay indefinitely at $E_{\text {crit }}$; they will spread out in energy in such a way as to eventually join the ambient Maxwellian population, doing so in the first instance via diffusion in velocity rather than systematic slowing down. A more complete treatment is needed to discuss the form of the photon spectrum and what it is telling us about flare fast electrons. McClements (1987) has included velocity diffusion effects, but only as one component of a complicated treatment which also features a number of other processes, and he does not explore the issues we address.

In this contribution we examine the evolution of injected electrons when the cold target assumption breaks down, in the slightly idealised case of a homogeneous source and constant background temperature. We include velocity diffusion as well as systematic slowing down, and reformulate the interpretation of observed photon spectra. The next Section formulates the problem and gives some analytical discussion. Section 3 illustrates our discussion with some calculated spectra, compared to RHESSI data. Section 4 gives brief conclusions.

\section{Velocity diffusion of fast electrons}

\subsection{Assumptions; Fokker-Planck equation}

In order to illustrate the consequences of velocity diffusion for photon spectra we consider an idealised problem in which all injected electrons thermalise in a uniform, homogeneous medium, characterised by a single, ambient electron density $n_{\mathrm{e}}$ and temperature $T_{\mathrm{e}}$. Loss at boundaries will have a negligible influence on the electron distribution function and pitchangle information will not be important for the calculation of the total, emergent photon spectrum, particularly since bremsstrahlung directionality is unimportant at the few $\mathrm{keV}$ photon energies appropriate here. We can gain significant insight, and also solve a problem appropriate to understanding the X-ray emission integrated over the whole of the event, by studying a steady-state situation, so no quantity depends on time. In practice $n_{\mathrm{e}}$ and $T_{\mathrm{e}}$ will evolve as a result of the thermal and hydrodynamic response of the atmosphere to the flare energy release, but this is a complication of detail rather than principle and we ignore it in the interests of gaining insight. (Relevant electron timescales such as the thermalisation time are at most on the order of seconds, whereas bulk changes to the plasma, such as changes of temperature as characterised by variation of the soft X-ray flux, take place over timescales of a few minutes.) Thus we can characterise the (pitch-angle integrated) electron distribution everywhere in the source by a single function $f(v)\left(\left(\mathrm{cm} \mathrm{s}^{-1}\right)^{-3}\right)$ of velocity $v\left(\mathrm{~cm} \mathrm{~s}^{-1}\right)$. The normalisation of $f$ is given by

$4 \pi \int_{0}^{\infty} f(v) v^{2} \mathrm{~d} v=N_{\mathrm{e}}$

where $N_{\mathrm{e}}$ is the total number of all electrons in the source.

An electron of $10 \mathrm{keV}$ initial energy will stop in a column depth of $2 \times 10^{19} \mathrm{~cm}^{-2}$ of fully ionised hydrogen, inside the coronal portion of a loop, e.g. for densities $>2 \times 10^{10} \mathrm{~cm}^{-3}$ and loop lengths $>10^{9} \mathrm{~cm}$, conditions not infrequently inferred in flares. Thermalisation, in the alternative case that this energy is close to the thermal speed, will occur in a comparable distance. In addition, magnetic field convergence may further enhance the coronal residence time of electrons and increase the effective distance available for thermalisation. Electrons well above thermal speeds will experience cold target conditions throughout the corona and chromosphere and will in any case be described correctly by what follows. Thus, while possibly not the case in all events, it is not unreasonable that all electrons for which finite thermal velocity ("warm") target effects are important thermalise in the coronal, warm target region.

This steady-state treatment will be valid as long as we use it on times that are not long enough for the injected electrons to become significant in number compared to the thermal distribution, but are longer than relaxation times for most injected electrons. Alternatively, we may regard it as giving the time integral of the distribution function in the case of an initial, impulsive injection, in which case the source function $S$ is actually the initial condition on $f$ (MacKinnon \& Craig 1991). The time integral of $f$ is the necessary quantity for calculation of the total bremsstrahlung photon yield.

We use the Fokker-Planck formalism for particle evolution under binary collisions (e.g. Rosenbluth et al. 1957; Montgomery \& Tidman 1964). We also make the assumption that the fast particles are "dilute", in the particular sense that they may be ignored in calculating the velocity space drift and diffusion coefficients: these may be evaluated purely from the background distribution. Then the steady-state, Fokker-Planck equation for $f(v)$, derived from Helander \& Sigmar (2002, pp. 37-38), may be written as

$-\frac{1}{v^{2}} \frac{\partial}{\partial v}\left(\left\{\Phi(v)-v \Phi^{\prime}(v)\right\}\left\{f+\frac{1}{2 v} \frac{\partial f}{\partial v}\right\}\right)=S(v)$,

where the function $\Phi$ is the error function:

$\Phi(x) \equiv \operatorname{erf}(x)=\frac{2}{\sqrt{\pi}} \int_{0}^{x} \mathrm{e}^{-y^{2}} \mathrm{~d} y$.

Here velocities $v$ have been normalised to the ambient electron thermal speed $v_{\mathrm{T}}=\sqrt{2 k T / m_{\mathrm{e}}}$, and times to the electron thermal collision time $t_{\mathrm{c}}=4 \pi \epsilon_{0}^{2} m_{\mathrm{e}}^{2} v_{\mathrm{T}}^{3} n_{\mathrm{e}}^{-1} \mathrm{e}^{-4}(\ln \Lambda)^{-1}$. Although 
there is no time-dependence in this problem, the electron injection function $S(v)$ is of course per unit time.

Equation (2) needs two boundary conditions. The boundary condition at infinity is: $(f+1 / 2 v \partial f / \partial v) \rightarrow 0$ as $v \rightarrow \infty$. This condition ensures that there is no flux of particles out of the system at infinity. For the other boundary condition we fix $f(v)$ at $v=0: f(0)=f_{0}$, consistent with the conditions for validity of our steady-state treatment and with the assumption of "diluteness" that justified the linearisation of the Fokker-Planck equation. $f_{0}$ describes the background thermal distribution. We integrate Eq. (2) once from $v$ to infinity and use the boundary condition at infinity, then again from 0 to $v$, employing an integrating factor $\mathrm{e}^{v^{2}}$ and using the boundary condition at 0 . Thus we find the solution

$f(v)=f_{0} \mathrm{e}^{-v^{2}}+2 \int_{0}^{v} \frac{v^{\prime} \mathrm{e}^{\left(v^{\prime 2}-v^{2}\right)}}{\Phi\left(v^{\prime}\right)-v^{\prime} \Phi^{\prime}\left(v^{\prime}\right)} \mathrm{d} v^{\prime} \int_{v^{\prime}}^{\infty} v^{\prime \prime 2} S\left(v^{\prime \prime}\right) \mathrm{d} v^{\prime \prime},(4)$ which will be used in Sect. 3 to calculate distributions and resultant photon spectra for various forms of $S$. The result (4) is rendered rather impenetrable, however, by the function $\Phi$. An illuminating, semi-quantitative analytical discussion may be carried out by invoking the large argument form of $\Phi(v)$, strictly applicable for $v \gg 1$, in which $\Phi(v)$ approaches unity for large $v$. In this case the Fokker-Planck equation becomes:

$$
-\frac{1}{v^{2}} \frac{\partial}{\partial v}\left(f+\frac{1}{2 v} \frac{\partial f}{\partial v}\right)=S(v)
$$

\subsection{Approximate solution}

First, we note that the LHS of Eq. (5) may be rewritten

$-\frac{1}{v^{2}}\left(1-\frac{1}{2 v^{2}}\right) \frac{\partial f}{\partial v}-\frac{1}{2 v^{3}} \frac{\partial^{2} f}{\partial v^{2}}$,

showing explicitly that the systematic rate of change of $v$ (the coefficient of $\partial f / \partial v$ ) does indeed display a zero, even in this approximate form, at $v=1 / \sqrt{2}$, quite close to the zero found using the full form of $\Phi$ by Emslie (2003). All the necessary qualitative features are included in the description of Eq. (5), in spite of its approximate nature, and its solutions will have the appropriate qualitative properties.

Note that the warm target corrections to the systematic slowing-down rate and the dispersive term both become important in the limit $v \rightarrow 1 / \sqrt{2}$. The arguments of Emslie (2003) rest on the presence of the zero (here at $v=1 / \sqrt{2}$ ) in the systematic slowing-down term. Electron slowing-down times approach $\infty$, so a finite emergent photon spectrum demands $S \rightarrow 0$ as $v \rightarrow 1 / \sqrt{2}$. However, in this limit the dispersive term has become important, removing the divergence in electron "lifetime". Using the boundary conditions as before, and changing the order of integration in the resulting integral, Eq. (5) has the solution

$$
\begin{aligned}
f(v)= & f_{0} \mathrm{e}^{-v^{2}}+\mathrm{e}^{-v^{2}} \int_{0}^{v} u^{2} S(u)\left(\mathrm{e}^{u^{2}}-1\right) \mathrm{d} u \\
& +\left(1-\mathrm{e}^{-v^{2}}\right) \int_{v}^{\infty} u^{2} S(u) \mathrm{d} u .
\end{aligned}
$$

In the absence of any source $S$, Eq. (5) has the background Maxwell-Boltzmann distribution $\left(f_{0} \mathrm{e}^{-v^{2}}\right)$ as its solution, as indeed does Eq. (2). The description in terms only of systematic slowing-down rates divorces the fast particle and background distributions. This is no longer the case in this diffusive treatment: the presence of the boundary condition at $v=0$, which must be satisfied using the same background density used to calculate the drift and diffusion coefficients, ensures that the fast particle distribution merges smoothly with the thermal "core". It follows that we are obliged to include also the contribution to photon emission from the thermal plasma, if we are indeed looking at photon energies such that velocity diffusion is important for the emitting electrons.

In the limit $v \rightarrow \infty$, Eq. (6) becomes

$f(v) \rightarrow \int_{v}^{\infty} u^{2} S(u) \mathrm{d} u$.

Recall that our $f$ is identical with the mean source electron distribution, the key quantity in interpreting X-ray emission (Brown et al. 2003). With the traditional assumptions of fast electrons slowing down in a cold, thick target, this mean distribution is just the cumulative distribution of the injected energy distribution. Equation (7) reproduces this result, as it should. We can rapidly recover well-known results in that limit, for instance Brown's (1971) relations between energy power-law spectral indices of observed photons and injected electrons.

We see that the three terms in the solution (6) consist of: the Maxwell-Boltzmann core of the distribution; a term which resembles the cold target result more and more closely as $v \rightarrow \infty$; and a term which forces these two regimes to merge smoothly.

\subsection{Mono-energetic injected population}

The special case of a mono-energetic form for $S$ is instructive:

$S(v)=\frac{S_{0} \delta\left(v-v_{0}\right)}{v^{2}}$

for some velocity $v_{0}$. Then the solution (6) becomes:

$f(v)= \begin{cases}\left\{f_{0}+S_{0}\left(\mathrm{e}^{v_{0}^{2}}-1\right)\right\} \mathrm{e}^{-v^{2}} & \text { if } v>v_{0} \\ f_{0} \mathrm{e}^{-v^{2}}+S_{0}\left(1-\mathrm{e}^{-v^{2}}\right) & \text { if } v<v_{0} .\end{cases}$

For $v<v_{0}$ the distribution is composed of the original, background Maxwellian distribution plus a component which is identical to the cold target result for $v, v_{0} \gg 1$, but which approaches 0 as $v \rightarrow 0$. This additional, non-Maxwellian component becomes less and less significant for smaller and smaller $v_{0}$. For $v>v_{0}$, the distribution is identical with the original background Maxwell-Boltzmann distribution, only with its normalisation increased. Since we must have $S_{0} \ll f_{0}$ for validity of the original linearisation of the Fokker-Planck equation, we see that the distribution will resemble the original Maxwellian more and more closely as $v_{0}$ gets closer and closer to 0 . This justifies the qualitative comments made in Sect. 1: electrons injected close to the thermal speed diffuse in energy rather than slowing down monotonically, merely adding their number to the original background Maxwellian. Figure 1 illustrates this. 


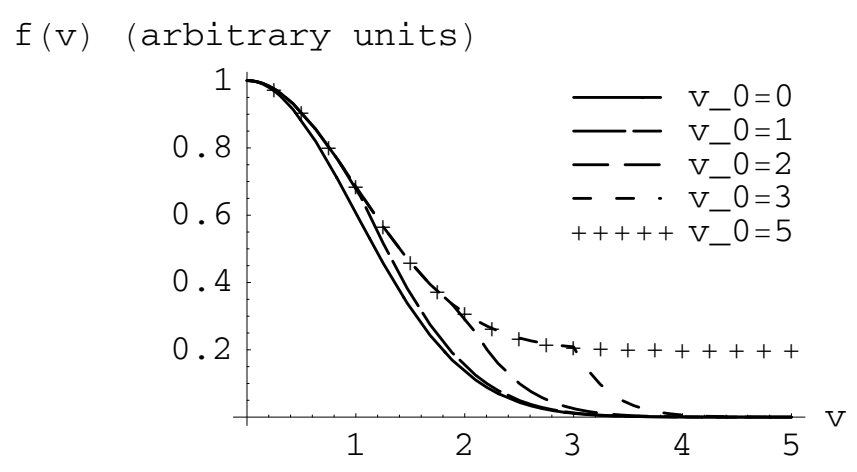

Fig. 1. Form of the relaxed, steady-state distribution resulting from injected, monoenergetic electrons. The different curves represent different values of the velocity (in units of the thermal speed) of the injected electrons. The noticeable change in behaviour between the curves for $v_{0}=2$ and $v_{0}=3$ highlights the injected electrons' transition from being in the thermalisation regime to being properly non-thermal.

\subsection{Deduction of $S$ for a power-law photon spectrum}

As mentioned in Sect. 1, the photon spectrum may in principle be inverted to yield the mean (source-averaged) electron distribution (Brown 1971; Brown et al. 2003). This is identical with the distribution function $f$ in the special case of our homogeneous source. If observations have given us $f$ in this way, Eqs. (2) or (5) then immediately give $S$. Consider the case of a power-law photon spectrum $I(\epsilon) \sim \epsilon^{-\gamma}$. Assume for the moment that this form holds at all photon energies of interest. Then the results of Brown (1971) give us $f(v) \sim v^{-2 \gamma-3}$. Inserting this form into Eq. (5) we find

$S(v) \sim(2 \gamma+3) v^{-2 \gamma-6}\left\{1-\frac{(2 \gamma+5)}{2} \frac{1}{v^{2}}\right\}$.

As in the case retaining only systematic energy loss (Emslie 2003), $S$ has a zero, changing sign at

$v_{*}=\sqrt{\gamma+5 / 2}$.

$S(v)$ takes negative values for $v<v_{*}$. We have seen that a diffusive treatment underlines the necessity of including the radiation from the thermal, "core" part of the distribution. In assuming the power-law photon spectrum to be appropriate at all photon energies we have implicitly neglected this contribution. $v_{*}$ represents the lowest velocity at which the single, uninterrupted power-law photon spectrum can still be reconciled with the presence of the Maxwell-Boltzmann core. Below $v_{*}$ we would have to actually remove particles from this core to prevent deviations from a power-law photon spectrum; hence $v_{*}$ 's dependence on $\gamma$. Moreover, we cannot "overcome" the core Maxwellian distribution by, for example, injecting a power-law energy distribution of electrons that persists down in energy towards thermal speeds. As we saw in Sect. 2.3, these electrons mostly thermalise diffusively, producing only a slight modification to the core.

We might follow Emslie (2003) and evaluate total electron energy content by integrating $S$, given by Eq. (10), from $v_{*}$ to $\infty$. Rather than adopting a lower energy cutoff for the power-law which evidently holds at high energies, however, this approach underlines the need for a consistent treatment of radiation from both thermal and accelerated electrons.

\section{Numerical illustrations and application to data}

\subsection{Numerical illustrations}

We return now to the full solution of the Fokker-Planck equation as given in Eq. (4), and provide some illustrative examples relevant to solar observations. We adopt as the source function $S(v)$ a power-law, employing the form

$$
\begin{aligned}
S(v) & =\tilde{S}(v) H\left(v-v_{0}\right) \\
& =S_{0}\left(\delta_{v}-3\right) v_{0}^{\delta_{v}-3} v^{-\delta_{v}} H\left(v-v_{0}\right),
\end{aligned}
$$

where $\tilde{S}$ is normalised such that per unit time (normalised to the electron thermal collision time) there are $S_{0}$ particles injected in total at velocities above $v_{0}$, and $S$ is prevented from going to infinity at low velocities by Heaviside's step function $H$ which removes all particles with velocities less than $v_{0}$.

For a homogeneous source, the emission rate of photons of energy $\epsilon$ per unit energy range per unit volume, $\mathrm{d} j / \mathrm{d} \epsilon$ (photons $\mathrm{s}^{-1} \mathrm{keV}^{-1} \mathrm{~cm}^{-3}$ ), may be found by multiplying the distribution function by $v \mathrm{~d} \sigma / \mathrm{d} \epsilon$ to obtain the instantaneous rate of emission of photons by electrons in the velocity range $\boldsymbol{v} \rightarrow \boldsymbol{v}+\mathrm{d} \boldsymbol{v}$, then integrating over all velocities (Brown 1971), noting that $\mathrm{d} v=4 \pi v^{2} \mathrm{~d} v$. This gives

$\frac{\mathrm{d} j}{\mathrm{~d} \epsilon}=n_{\mathrm{e}} v_{\mathrm{T}}^{3} \int_{\sqrt{\epsilon / k T}}^{\infty} f(v) \frac{\mathrm{d} \sigma}{\mathrm{d} \epsilon} v^{3} \mathrm{~d} v$,

where $n_{\mathrm{e}}$ is the background plasma number density and $\frac{\mathrm{d} \sigma}{\mathrm{d} \epsilon}$ is the Bethe-Heitler cross-section:

$\frac{\mathrm{d} \sigma}{\mathrm{d} \epsilon}=\frac{Q_{0} m_{\mathrm{e}} c^{2}}{\epsilon E} \ln \left(\frac{1+\sqrt{1-\epsilon / E}}{1-\sqrt{1-\epsilon / E}}\right)$.

Here, $E$ is the electron kinetic energy and $Q_{0}$ is given by

$Q_{0}=\frac{8}{3} \alpha r_{\mathrm{e}}^{2}$,

where the fine structure constant $\alpha \approx 1 / 137$ and $r_{\mathrm{e}}=2.82 \times$ $10^{-13} \mathrm{~cm}$ is the classical electron radius. The photon spectrum, $\mathrm{d} \tilde{j} / \mathrm{d} \epsilon$ (photons $\mathrm{s}^{-1} \mathrm{~cm}^{-2} \mathrm{keV}^{-1}$ ), that would be observed by RHESSI is given by

$\frac{\mathrm{d} \tilde{j}}{\mathrm{~d} \epsilon}=\frac{V}{4 \pi r_{\oplus}^{2}} \frac{\mathrm{d} j}{\mathrm{~d} \epsilon}$,

where $V$ is the volume of the source and $r_{\oplus}$ is the distance from the Sun to the Earth. Since radiation from the whole emitting volume is observed, the value of $V$ will be determined implicitly by the spectral fitting process (see Sect. 3.2) and need not be separately evaluated.

As previously stated, the presence of $\Phi$ in Eq. (4) renders a full analytical solution intractable. Therefore we proceed numerically, using Romberg integration (Press et al. 1992) to evaluate Eq. (12) with $f$ given by Eq. (4).

The parameters which may be varied in the numerical simulations are: the ratio of $f_{0}$ to $S_{0}$ i.e. the relative magnitudes of 


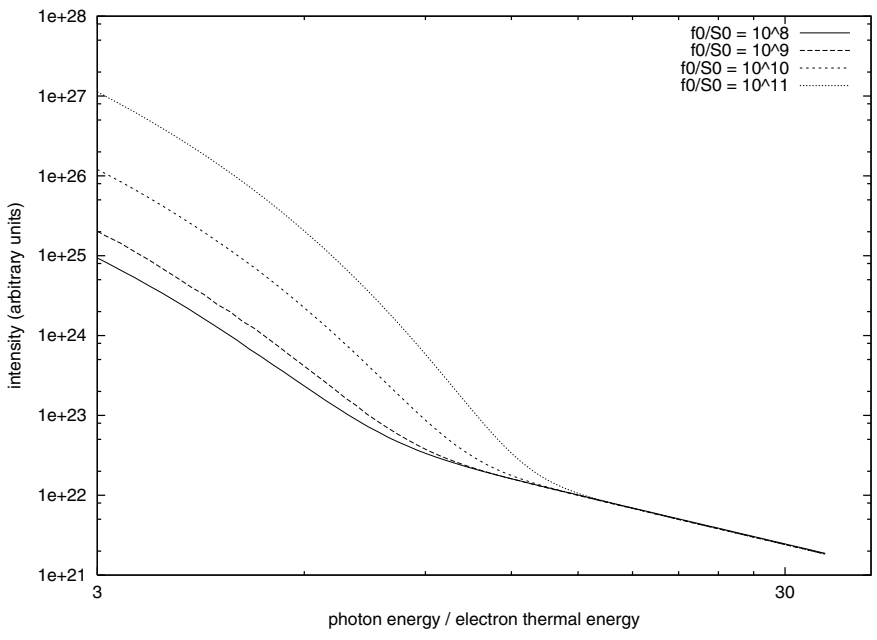

Fig. 2. Simulated $X$-ray photon spectra, showing the effect of altering the ratio of $f_{0}$ to $S_{0}$. The photon energy is normalised to the kinetic energy of an electron of thermal velocity. The emitted intensity values are arbitrary, since we are interested only in relative changes to the spectral profiles.

the background Maxwellian population and the injected powerlaw electrons; the lower cutoff velocity of the injected electrons, $v_{0}$; and the spectral index $\delta$ of the power-law. It should be noted that in the majority of the literature, the power-law used to model flare electrons is a power-law in energy of the form $S(E)=S_{0} E^{-\delta}$. For consistency and ease of comparison we shall refer to this $\delta$ in subsequent discussion. The corresponding $\delta_{v}$ for our velocity power-law, Eq. (11), is related to $\delta$ by the expression $\delta_{v}=2 \delta-1$. Unless otherwise stated, the default values of the parameters are $f_{0} / S_{0}=10^{8}, \delta=4.0$, and $v_{0}=v_{\mathrm{T}}$.

Figure 2 illustrates the effect of altering the ratio $f_{0} / S_{0}$. The logarithmically-plotted photon spectra consist of two main regions: a straight power-law profile at high photon energy, blending smoothly into a Maxwellian profile at lower energy. As would be expected, increasing the relative contribution of the Maxwellian background has no effect at high photon energies since here the profile only contains contributions from electrons of the photon energy or higher. However, a larger $f_{0} / S_{0}$ value leads to a correspondingly higher contribution to the Maxwellian portion of the spectra from the background plasma. Furthermore, this larger value also corresponds to an increase in the photon energy up to which the Maxwellian impinges on the otherwise straight power-law: the profile departs from the straight portion at higher energy for a larger $f_{0} / S_{0}$.

The alteration of the electron energy spectral index $\delta$ is depicted in Fig. 3. As may be seen, this has minimal effect at low photon energy, but a larger $\delta$ results in a correspondingly steeper slope in the power-law region of the spectrum. A greater value for $\delta$ also causes a more rapid reduction in the total number of electrons as a function of increasing energy in the injected population. Thus, a higher $\delta$ leads to a relative reduction in the intensity of the power-law spectrum for a given photon energy. This reduced contribution from the power-law electrons also increases the photon energy up to which the Maxwellian element forms a significant part of the resultant

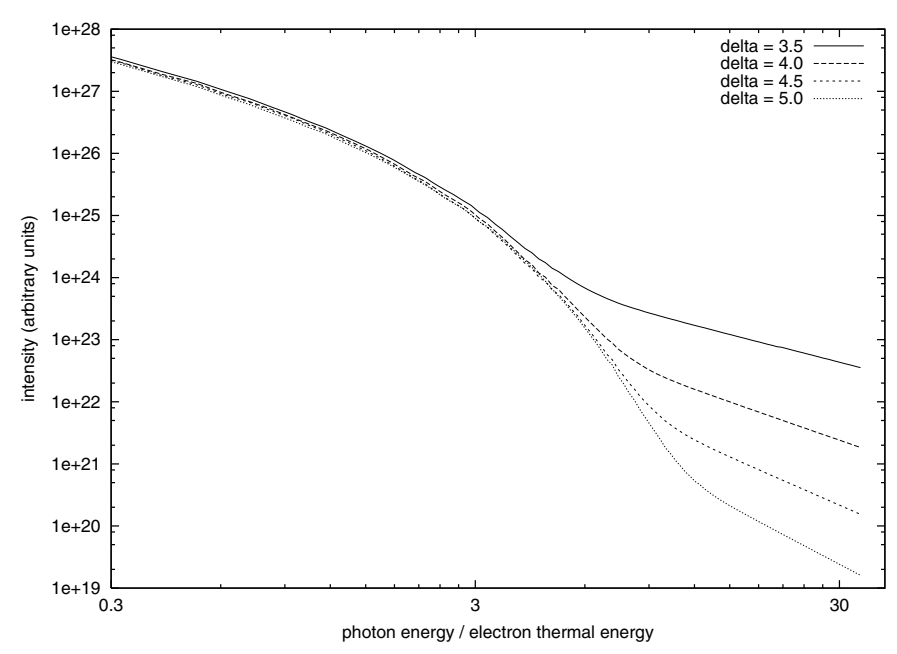

Fig. 3. As Fig. 2, but with variation of the power-law spectral index $\delta$, and over an expanded range of photon energy.

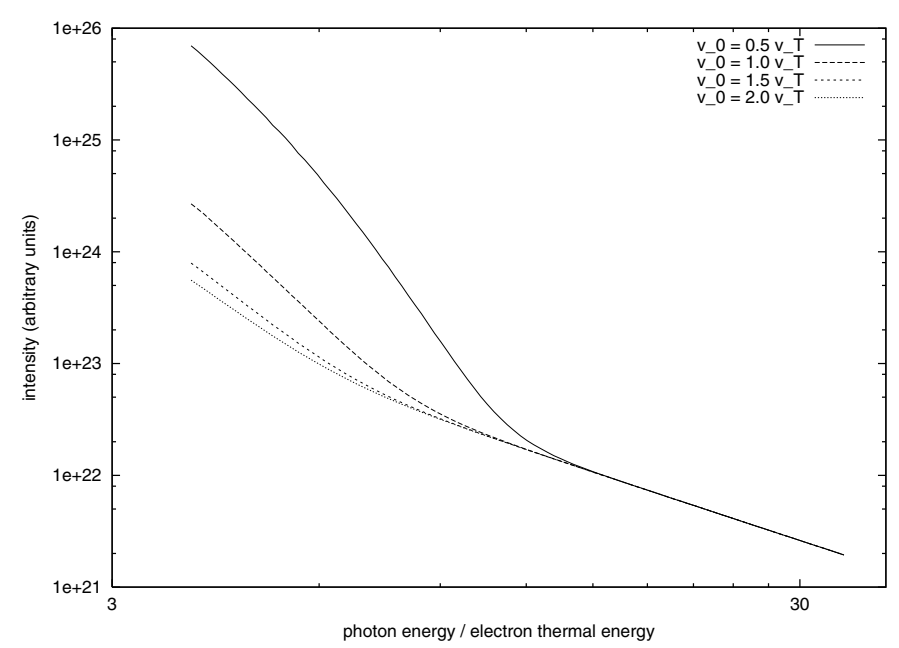

Fig. 4. As Fig. 2, but with variation of the power-law cutoff $v_{0}$.

profile. Consequently, the departure from the straight powerlaw profile occurs at a higher photon energy for larger $\delta$ values, visually mimicking a non-existent change in the power-law low energy cutoff.

Actual variation of the cutoff, $v_{0}$, is shown in Fig. 4. Since electrons injected below a few times $v_{\mathrm{T}}$ thermalise rather than slowing down systematically, allowing the cutoff to extend to lower energies merely adds electrons to the "background" Maxwell-Boltzmann distribution. This explains the counterintuitive result, clearly visible in Fig. 4, that a lower value of $v_{0}$ results in a spectrum which attains power-law form at higher photon energies: the large number of injected electrons at low energies thermalise and enhance the Maxwell-Boltzmann distribution, concealing the lower-energy portion of the power-law form.

\subsection{Comparisons to RHESSI data}

The recent launch of the Reuven Ramaty High Energy Solar Spectroscopic Imager (RHESSI) has opened a new era in high resolution X-ray spectroscopy of solar flares 
(Lin et al. 2002). The analysis of solar flare spectra has revealed statistically significant deviations from a simple isothermal and power-law model (Kontar et al. 2003). The detailed analysis of the X-ray producing spectra using a model-independent inversion technique (Piana et al. 2003) shows deviations from the pure isothermal model. These new findings can be treated as the manifestation of velocity space diffusion in warm target plasma.

For illustrative purposes, we consider a few example events with sufficiently high count rate to provide reliable photon statistics. We have limited our analysis to within the energy range $10-50 \mathrm{keV}$, where thermal and nonthermal components merge. Below $9 \mathrm{keV}$, the bremsstrahlung continuum is contaminated by a complex of strong iron lines. Above $\sim 50 \mathrm{keV}$, spectral features not related to the model discussed become dominant (Kontar et al. 2003).

We fit the model spectra to the observed spectra by optimising over 4 parameters: the relative magnitudes of the background and injected electron populations, $f_{0} / S_{0}$; the injected electron power-law low-energy cutoff, $v_{0}$; the background temperature, $T_{\mathrm{e}}$; and the injected electron power-law spectral in$\operatorname{dex}, \delta$. The optimisation seeks to minimise an un-normalised $\chi^{2}$ fit statistic - in this case absolute values of $\chi^{2}$ must be treated with caution since the process of deconvolving the RHESSI instrument response from the observed counts spectrum to produce the photon spectrum introduces an element of error on each photon spectrum data point which is difficult to quantify precisely (Smith et al. 2002). However, we only compare relative values of the fit statistic to optimise the model fits, so un-normalised $\chi^{2}$ is sufficient for our purpose. As may be seen from Fig. 5, sets of optimal model parameters may be found which give model spectra that closely match the observed RHESSI spectra. The model parameters corresponding to the smallest fit statistic in each case are given in Table 1.

As previously discussed, our steady-state treatment is valid on timescales longer than the collisional timescale but shorter than the timescale for changes in the temperature of the flaring loop. Thus, the fitted model parameters describe the flare plasma at any instant, but will change with time as the plasma evolves during the flare. To obtain a simple estimate of the energy content of the electrons in the example flares, we fit a model spectrum to an observed RHESSI spectrum from during the impulsive phase, and multiply the instantaneous energy content by the duration of the phase. To obtain the instantaneous energy, we insert the optimal fitted values of the relevant parameters into the source function, Eq. (11), and calculate the total energy content of the fast electrons by integrating the electron kinetic energy over all possible electron velocities:

$E_{\text {fast }}=\int_{v_{0}}^{\infty} \frac{1}{2} m_{\mathrm{e}} v^{2} S(v) v^{2} \mathrm{~d} v$.

The electron energy content of the thermal background plasma may also be obtained from the optimal fitted parameters:

$E_{\text {therm }}=\frac{3}{2} N k T_{\mathrm{e}}=\frac{3}{2}\left(f_{0} v_{\mathrm{T}}^{2}\right) k T_{\mathrm{e}}$.

(For our present illustrative purposes, we assume a background plasma density of $10^{15} \mathrm{~m}^{-3}$ - typical of the lower corona - to
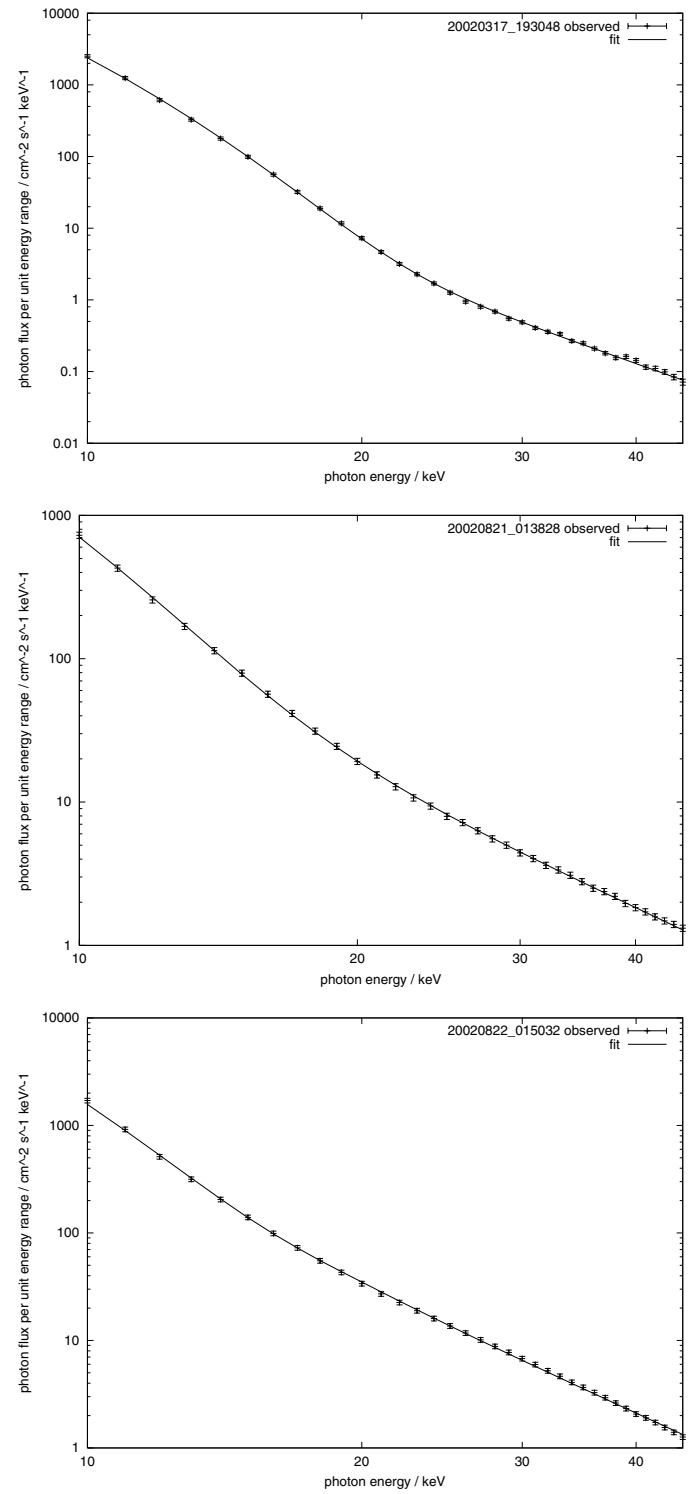

Fig. 5. X-ray photon spectra recorded by RHESSI during the flares of 17th March 2002 at 19:30:48 UT, 21st August 2002 at 01:38:28 UT, and 22nd August 2002 at 01:50:32 UT. Model fits to the observed spectra are shown as solid lines.

obtain the absolute value of $f_{0}$ from the value of the emission measure as determined from the fits.) The total energy in all the electrons is the sum of $E_{\text {fast }}$ and $E_{\text {therm }}$.

We find single values of the source parameters to represent the whole of the data time interval. The main assumption in doing this is that background parameters $\left(f_{0}, T_{\mathrm{e}}\right)$ do not change. If this is the case then interpreting the time integral of the data gives us the same result as integrating a temporal sequence of data fits (as mentioned in Sect. 2; see also MacKinnon \& Craig 1991). Although these parameters may change, this will be partly because of the relaxation of the fast electrons. Qualitatively, the procedure here may overestimate the injected electron distribution, because temperature will increase, and thus more of the observed photon spectrum will be due to "thermal" electrons, as time goes on. To address this issue quantitatively we would have to drop the linearisation of 
Table 1. Optimal fit parameters for our model fits to RHESSI data.

\begin{tabular}{cccccccc}
\hline \hline Flare & $f_{0} / S_{0}$ & $v_{0}$ & $T_{\mathrm{e}}(\mathrm{MK})$ & $\delta$ & $E_{\text {fast }}(\mathrm{J})$ & $E_{\text {therm }}(\mathrm{J})$ & $E_{\text {tot }}(\mathrm{J})$ \\
\hline $2002 / 03 / 17$ & $1.46 \times 10^{7}$ & 1.18 & 21.2 & 6.30 & $3.79 \times 10^{25}$ & $1.43 \times 10^{24}$ & $3.94 \times 10^{25}$ \\
$2002 / 08 / 21$ & $3.40 \times 10^{7}$ & 2.66 & 29.7 & 4.86 & $1.47 \times 10^{23}$ & $1.38 \times 10^{23}$ & $2.84 \times 10^{23}$ \\
$2002 / 08 / 22$ & $5.28 \times 10^{6}$ & 2.90 & 25.0 & 5.69 & $3.75 \times 10^{23}$ & $5.32 \times 10^{23}$ & $9.07 \times 10^{23}$ \\
\hline
\end{tabular}

$E_{\text {fast }}$ and $E_{\text {therm }}$ are the energy contents of the fast and background electrons respectively, calculated using the optimal parameters given. $E_{\text {tot }}$ is the total energy content of all the electrons.

Table 2. Optimal fit parameters for fits to RHESSI data using a simple thermal plus power-law model.

\begin{tabular}{cccccccc}
\hline \hline Flare & $f_{0} / S_{0}$ & $v_{0}$ & $T_{\mathrm{e}}(\mathrm{MK})$ & $\delta$ & $E_{\text {fast }}(\mathrm{J})$ & $E_{\text {therm }}(\mathrm{J})$ & $E_{\text {tot }}(\mathrm{J})$ \\
\hline $2002 / 03 / 17$ & $2.52 \times 10^{8}$ & 3.10 & 22.0 & 6.41 & $2.68 \times 10^{21}$ & $1.44 \times 10^{24}$ & $1.45 \times 10^{24}$ \\
$2002 / 08 / 21$ & $3.98 \times 10^{8}$ & 1.93 & 29.8 & 4.98 & $8.62 \times 10^{21}$ & $1.53 \times 10^{23}$ & $1.61 \times 10^{23}$ \\
$2002 / 08 / 22$ & $1.11 \times 10^{7}$ & 3.16 & 25.2 & 5.91 & $6.73 \times 10^{22}$ & $5.37 \times 10^{23}$ & $6.05 \times 10^{23}$ \\
\hline
\end{tabular}

$E_{\text {fast }}$ and $E_{\text {therm }}$ are the energy contents of the fast and background electrons respectively, calculated using the optimal parameters given. $E_{\text {tot }}$ is the total energy content of all the electrons.

the Fokker-Planck equation, Eq. (2), resulting in a considerably more complex problem that we do not address here. Such a fuller treatment would also allow us to precisely determine the realm of validity of our linearisation.

The time intervals we use include the bulk of the hard X-ray emission from the flares in question, but of course a more complete discussion of flare energetics would also integrate over the entire history of the flare.

Also given in Table 1 are the total energy contents of all electrons and of the injected and background electron populations individually for each event studied. The flares of $21 \mathrm{st}$ and 22nd August 2002 have comparable energies in the thermal and fast components, and a total energy consistent with an $\mathrm{M}$ class flare. Both also have low-energy cutoffs at only a few times the thermal speed, indicating that velocity diffusion will be relevant. The optimal fitted parameters for the flare of 17th March 2002 result in a much larger total energy which would correspond to a larger and more energetic flare. However, in this case the calculated energy content of the injected electrons is many times greater than that of the background thermal electrons. This arises because the fitted low-energy cutoff is very close to the thermal speed itself, and the power-law spectral index is very large, meaning that the injected population will have a huge number of electrons very near to the thermal speed. These will rapidly thermalise and give rise to the bulk of the Maxwellian portion of the photon spectrum as shown in Fig. 5, dominating the emission from the background plasma. While this set of model parameters corresponds to a minimum fit statistic, and produces a model spectrum which closely reproduces the observations, they also imply a situation where the injected electrons are no longer "dilute" and our linearisation is no longer applicable.

Lin et al. (2003) employed Emslie's (2003) formulae for flare electron energy content in their analysis of the 23rd July 2002 flare. This analysis assumed that the injected electrons had a power-law low-energy cutoff at approximately the thermal speed ( $T \approx 23 \mathrm{MK}$ ) and, unlike our treatment, does not account for any thermalisation of these lower energy injected electrons. Lin et al. found the fast electron energy content for this X4.8 flare to be in excess of $10^{27} \mathrm{~J}$. This is very much greater than the highest total energies ever deduced for the largest flares. We also predict very large energies in the injected electrons for cases where $v_{0}$ is very low. However, our estimates are not as extremely high as those made using Emslie's formulae, since our treatment includes the appropriate velocity diffusion effects for the lower energy injected electrons.

Due to the unambiguous nature of the straight portion of the spectral profile at high energies, the fitted value of $\delta$ is well-constrained. However, as is evident from Figs. 2 and 4, variation of the values of $f_{0} / S_{0}$ and $v_{0}$ lead to similar variations in the shape of the resulting spectral profile. This suggests the possibility of a degeneracy in the fitted values of these parameters, which is indeed the case. The quoted value of the fitted $v_{0}$ given in the table is that for the most optimal fit. However, it was found that the value of $v_{0}$ could be varied from around one half to two times the optimal value for only a $10 \%$ increase in the value of the fit statistic. Therefore we are reluctantly forced to conclude that the value of $v_{0}$ is less wellconstrained by the data than Emslie's original argument might suggest. Similarly, $f_{0} / S_{0}$, and to a lesser extent $T_{\mathrm{e}}$, cannot be unambiguously determined. The value for the total electron energy varies by around an order of magnitude when the value of $v_{0}$ is varied over our selected $10 \%$ range of fit statistic acceptability, with the total energy decreasing as $v_{0}$ is increased. Thus, while the model can reproduce observed photon spectra, its nature may preclude a precise determination of the flare electron energy content.

For comparative purposes, Table 2 gives fitting parameters as derived from fits using a "simple" thermal plus power-law model, as may be employed in e.g. the OSPEX package in the standard RHESSI analysis software (e.g. Schwartz et al. 2002). As measured by our fit statistic, these fits are statistically acceptable at a similar confidence level to our model fits. As may be seen, the simple fits give consistently larger 
values of $f_{0} / S_{0}$ (and comparable or slightly greater temperatures) than our model fits, since all of the thermal part of the spectrum must be accounted for by the Maxwellian background with no contribution from thermalising fast electrons. This results in higher energy contents for the thermal background electrons, but the total energy contents are lower than for our fits since the thermalisation process is an energetically expensive way of producing "thermal" photons. The simple fit values for the low-energy cutoffs are comparable with those of our model in that they are a few times the thermal speed, but again this parameter is not well-constrained. The largest difference is for the 17th March flare, for which our model suggested an extremely low $v_{0}$. The simple fit is more conservative, and consequently does not give the very large energy content in the injected electrons as derived from our model fits.

\section{Conclusions}

We have seen that for the case of accelerated flare electrons impinging on a warm target, the effects of velocity diffusion should not be neglected. The process by which the lower energy electrons of the injected population thermalise and merge with the ambient thermal background is of importance when describing the behaviour of the electrons in this regime. In particular, it has the effect of "smearing out" this region of the resulting bremsstrahlung photon spectrum, which in many cases is therefore not well described by a simple isothermal and power-law model. However, this region can be modelled effectively by a treatment including velocity diffusion effects.

A consequence of including velocity diffusion in the analysis is that simple interpretation of observed photon spectra can be deceptive. For example, as has been shown in Figs. 2-4, determining the photon energy down to which the spectrum remains power-law-like does not allow a simple evaluation of the parameters of the injected electron population, most particularly its low energy cutoff. This in turn hinders determination of the flare electron energy content.

The surprising behaviour of the photon spectra is highlighted by the fits to RHESSI data in Fig. 5: visual inspection of the spectra would seem to suggest that the 17th March flare should have the highest temperature background plasma, since the Maxwellian portion of the spectrum is more prominent and extends to higher photon energy in this flare than in the others considered. However, the fitted parameters imply that the 17th March flare actually has the lowest background plasma temperature. The form and extent of the Maxwellian component of the photon spectrum in this case is completely dominated by thermalising electrons from the lower energies of the injected population. This result emphasises that the thermalising process effectively couples the injected and the background populations, such that their contributions to the overall photon spectrum cannot easily be separated. In effect, the distinction between the background and injected populations becomes rather arbitrary at these low energies, and it is no longer meaningful to distinguish between a background thermal electron and an electron which has thermalised out of the injected population. Further, because of this strong coupling it is also not possible to "swamp" the thermal region of the photon spectrum by contriving a large injected power-law population with a very low cutoff energy, as the lowest energy electrons will inevitably thermalise. However, this thermalisation process is an energetically expensive way to produce thermal emission.

The steady-state solution presented here has illuminated many of the consequences of velocity diffusion in the context of solar flares. However, an approach which explicitly accounts for time-dependence would be an interesting further development. This would allow modelling of the evolution of the plasma parameters over the duration of a flare, and may also be of benefit for more precise evaluations of the flare energy budget.

Acknowledgements. We thank the referee, Dr. N. Vilmer, for helpful comments which improved the paper. The work by P.H. was funded by the UK Physical Sciences and Engineering Research Council. R.K.G. is supported by a UK Particle Physics and Astronomy Research Council CASE Award.

\section{References}

Brown, J. C. 1971, Sol. Phys., 18, 489

Brown, J. C., Emslie, A. G., \& Kontar, E. P. 2003, ApJ, 595, L115

Emslie, A. G. 2003, ApJ, 595, L119

Emslie, A. G., Kontar, E. P., Krucker, S., \& Lin, R. P. 2003, ApJ, 595, L107

Helander, P., \& Sigmar, D. J. 2002, Collisional Transport in Magnetized Plasmas (Cambridge University Press)

Hoyng, P., Brown, J. C., \& van Beek, H. F. 1976, Sol. Phys., 48, 197

Kane, S. R., McTiernan, J., Loran, J., et al. 1992, ApJ, 390, 687

Kontar, E. P., Brown, J. C., Emslie, A. G., et al. 2003, ApJ, 595, L123

Lin, R. P., \& Hudson, H. S. 1976, Sol. Phys., 50, 153

Lin, R. P., Dennis, B. R., Hurford, G. J., et al. 2002, Sol. Phys., 210, 3

Lin, R. P., Krucker, S., Hurford, G. J., et al. 2003, ApJ, 595, L69

MacKinnon, A. L., \& Craig, I. J. D. 1991, A\&A, 251, 693

McClements, K. G. 1987, A\&A, 175, 255

Montgomery, D. C., \& Tidman, D. A. 1964, Plasma Kinetic Theory (McGraw-Hill)

Piana, M., Massone, A. M., Kontar, E. P., et al. 2003, ApJ, 595, L127

Press, W. H., Teukolsky, S. A., Vetterling, W. T., \& Flannery, B. P. 1992, Numerical Recipes in C (Cambridge University Press)

Rosenbluth, M. N., MacDonald, W. M., \& Judd, D. L. 1957, Phys. Rev., 107, 1

Saint-Hilaire, P., \& Benz, A. O. 2005, A\&A, 435, 743

Schwartz, R. A., Csillaghy, R. A., Tolbert, A. K., et al. 2002, Sol. Phys., 210, 165S

Smith, D. M., Lin, R. P., Turin, P., et al. 2002, Sol. Phys., 210, 33

Vilmer, N., \& MacKinnon, A. L. 2003, in Energy Conversion and Particle Acceleration in the Solar Corona, ed. K.-L. Klein (Springer), 127-160 